Apidologie, 1985, 16 (1), 19-30

\title{
ZUR BESTIMMUNG VON DIASTASE, INVERTASE UND HMF IN HONIG
}

Jost H. DUSTMANN, Jacob P. van PRAAGH, Katharina BOTE

Niedersächsisches Landesinstitut für Bienenforschung, Wehlstr. 4a D-31 Celle

\section{ZUSAMMENFASSUNG}

Um die Naturbelassenheit eines Honigs zu bestimmen, insbesondere Wärme- und Lagerschäden zu erfassen, sind mehrere chemische Analysenmethoden üblich, deren Genauigkeit und Zeitaufwand in vorliegender Arbeit untersucht werden. Hierbei zeigte sich, dass die Messung der SaccharaseAktivität (Invertase, $\alpha$-Glucosidase) in der Methode nach SiEgenthaler deutliche Vorteile gegenüber der gebräuchlichen Bestimmung des Gehaltes an Hydroxymethylfurfural (HMF) nach WiNKLER aufweist : die Genauigkeit und Reproduzierbarkeit der Saccharase sind grösser, der Umgang mit dem hochgiftigen Reagenz Toluidin entfällt, und der Zeitaufwand ist für beide Tests gleich. $\mathrm{Da}$ das Mass an Saccharase-Aktivität ohnehin einen besseren Indikator für die Naturbelassenheit eines Honigs darstellt als der HMF-Gehalt, wird vorgeschlagen, im Rahmen der Honigqualitätsprüfung als erstes Kriterium für Naturbelassenheit die Saccharase-Aktivität nach der Methode Siegenthaler zu messen. Der HMF-Test sollte lediglich bei von Natur aus enzymschwachen Honigen eingesetzt werden.

Zwischen den Invertase-Bestimmungen nach SiegentHaler in U/kg und nach Gontarski (in SZ) besteht eine lineare Beziehung. Eine Umrechnung der jeweiligen Einheiten ist somit möglich.

\section{EINLEITUNG}

Bei Qualitätsprüfungen von Honig werden als Nachweis für Wärme- und Lagereinflüsse bzw. für das Ausmass am Naturbelassenheit die Honigenzyme Diastase ( $\alpha$-Amylase) und Invertase (Saccharase, $\alpha$-Glucosidase), sowie das Zuckerabbauprodukt HMF (Hydroxymethylfurfural) gemessen.

Die Diastase-Bestimmung erfolgt lebensmittelrechtlich gemäss EG-Richtlinien vom 27.7.74 nach der Methode SCHADE et al. (1958). Zahlreiche Autoren haben darauf hingewiesen, dass das Enzym Diastase als Indikator für Wärme- und Lagerschäden wenig geeignet ist (GonTARsKı, 1961 ; HADORn et al., 1962 ; White et al., 1964). Weit besseren Aufschluss hierüber ergibt das Enzym Invertase. Für weitgehend naturbelassene Honige liegt die Invertaseaktivität (Saccharasezahl SZ) 
in der Regel über 10 Einheiten (SZ > 10) (Dustmann, 1967). Die in der Untersuchungspraxis bisher gebräuchlichen Methoden sind mit Nachteilen verbunden :

Die Messung der Diastase nach Schade ist abgesehen von der geringen Spezifität sehr zeitaufwendig. Es wurde daher nach anderen Verfahren gesucht (z.B. Gontarski, 1954). Kerkvliet und V.D. Putten (1973) weisen darauf hin, dass je nach Vorschriftsvariante Unterschiede in der Diastasezahl auftreten können, die um den Faktor 2 differieren. Ein von uns durchgeführter kleiner Ringversuch zeigt ähnliche Ergebnisse (Tab. 1).

TAB. 1. - Ringversuch, Diastase-Bestimmung (nach Schade)

TABL. 1. - Determination of Diastase (after ScHade)

\begin{tabular}{c|c|c|c|c|c}
\hline $\begin{array}{c}\text { Probe } \\
\text { Nr. } \\
\text { Sample No. }\end{array}$ & $\begin{array}{c}\text { Labor 1 } \\
\text { Lab. 1 } \\
\text { DZs }\end{array}$ & $\begin{array}{c}\text { Labor 2 } \\
\text { Lab. 2 } \\
\text { DZs }\end{array}$ & $\begin{array}{c}\text { Labor 3 } \\
\text { Lab. 3 } \\
\text { DZs }\end{array}$ & $\begin{array}{c}\text { Labor 4 } \\
\text { Lab. 4 } \\
\text { DZs }\end{array}$ & $\begin{array}{c}\text { PZllen-Analyse } \\
\text { Pollen analysis }\end{array}$ \\
\hline A 1 & 11,3 & 29,4 & 14,9 & 8,6 & $\begin{array}{c}\text { Osteuropa : Mischtracht } \\
\text { Eastern Europe : mixed flow }\end{array}$ \\
\hline A 2 & 13,7 & 5,5 & 2,9 & $\begin{array}{c}\text { Deutschland : Kreuzblütler + Honigtau } \\
\text { Germany : Crucifers + Honeydew }\end{array}$ \\
\hline A 3 & 18,5 & 50,0 & 24,7 & 15,4 & $\begin{array}{c}\text { Ubersee : Mischtracht } \\
\text { Overseas : mixed flow }\end{array}$ \\
\hline
\end{tabular}

Das Gontarski-Verfahren für die Invertase-Messung ist ebenfalls mit sehr grossem Zeitaufwand verbunden. Um diesen Schwierigkeiten im Routinebetrieb auszuweichen, wird in der Untersuchungspraxis mehrerer EG-Staaten die HMFBestimmung nach WinKLER (1955) für die Honigqualitätskontrolle angewandt. Diese Bestimmung verläuft zwar recht schnell, eines der zu verwendenden Reagentien (p-Toluidin) ist jedoch sehr giftig. Nach der deutschen Verordnung über gefährliche Arbeitsstoffe von 29. Juli 1980 ist p-Toluidin entsprechend R23/24/25-33 ; S28-36/37-44 einzustufen. Das bedeutet : Arbeiten mit Schutzkleidung und Handschuhen sowie unter einem Abzug.

Diese hier aufgeführten Probleme haben uns bewogen, die jetzige Honiganalysen-Praxis neu zu überdenken. Es gibt Analysen-Verfahren, die die Möglichkeit schaffen, die erwähnten Probleme zu beseitigen. Wir haben einerseits die Diastase-Bestimmung nach SCHADE mit der nach GonTARSKI verglichen und andererseits einen Vergleich zwischen den Invertase-Bestimmungen nach GoNTARSKI und nach Siegenthaler (1977) durchgeführt. 
Die Auswahl einer bestimmten Analysenmethodik sollte nicht ohne Abschätzung der Reproduzierbarkeit erfolgen. Deshalb haben wir sowohl für die HMF- als auch für die Invertase-Bestimmung die Genauigkeit statistisch überprüft.

\section{METHODIK UND MATERIAL}

\subsection{Die Honige}

Für die Analysen wurden die in der Tabelle 2 aufgeführten Honige eingesetzt, deren Trachtherkunft und Zusammensetzung grosse Unterschiede aufweisen.

\subsection{Diastase-Bestimmung}

Die Diastase-Bestimmung nach SCHADE wurde nach den im Schweizerischen Lebensmittelbuch veröffentlichten Richtlinien [nach Schade, MARSH und ECKERT (1958), modifizierte Methode nach Hadorn (1961)] durchgeführt. Als lösliche Stäıke wurde Merck 1252 verwendet. Die Messung der Transmission erfolgte mit einem Zeiss Photometer PMQII bei $575 \mathrm{~nm}$ ( $2 \mathrm{~cm}$ Küvetten).

Für die Diastase-Bestimmung nach GoNTARSKI (1954) diente ebenfalls das o.a. Stärkefabrikat. Als Photometer wurde ein Zeiss PM6 bei $570 \mathrm{~nm}$ eingesetzt (1 cm Küvetten).

\subsection{Invertase-Bestimmung}

Hier wurde die reduktometrische Methode nach GonTarski (1957) und die spektralphotometrische Erfassung von p-Nitrophenol nach Siegenthaler (1977) angewandt (Zeiss PM6, 400 nm, $1 \mathrm{~cm}$ Küvetten).

\subsection{HMF-Bestimmung}

Die Bestimmung des HMF-Gehaltes erfolgte nach der üblichen Methode WinkLER (1955), Als Toluidin-Reagenz diente das Fabrikat Merck Nr. 808315. Die Extinktionsmessung crfolgte mit PM6 bei $550 \mathrm{~nm}$ in $1 \mathrm{~cm}$ Küvetten.

\subsection{Slatistik}

Die Berechnung der Regressionsgeraden erfolgte mittels der Methode der klcinsten Quadrate.

\section{ERGEBNISSE}

\subsection{Diastase}

Wie aus der Abbildung $1 \mathrm{zu}$ ersehen ist, zeigt ein Vergleich zwischen den Diastase-Bestimmungen nach GonTarsKı und Schade, dass eine statistisch zuverlässige lineare Abhängigkeit zwischen den beiden Analysen-Verfahren besteht, Für unsere Analysen ergibt sich somit folgende Beziehung :

Diast. (n. Schade) $=0.2162$ Diast. (n. Gont.) -2.0752 


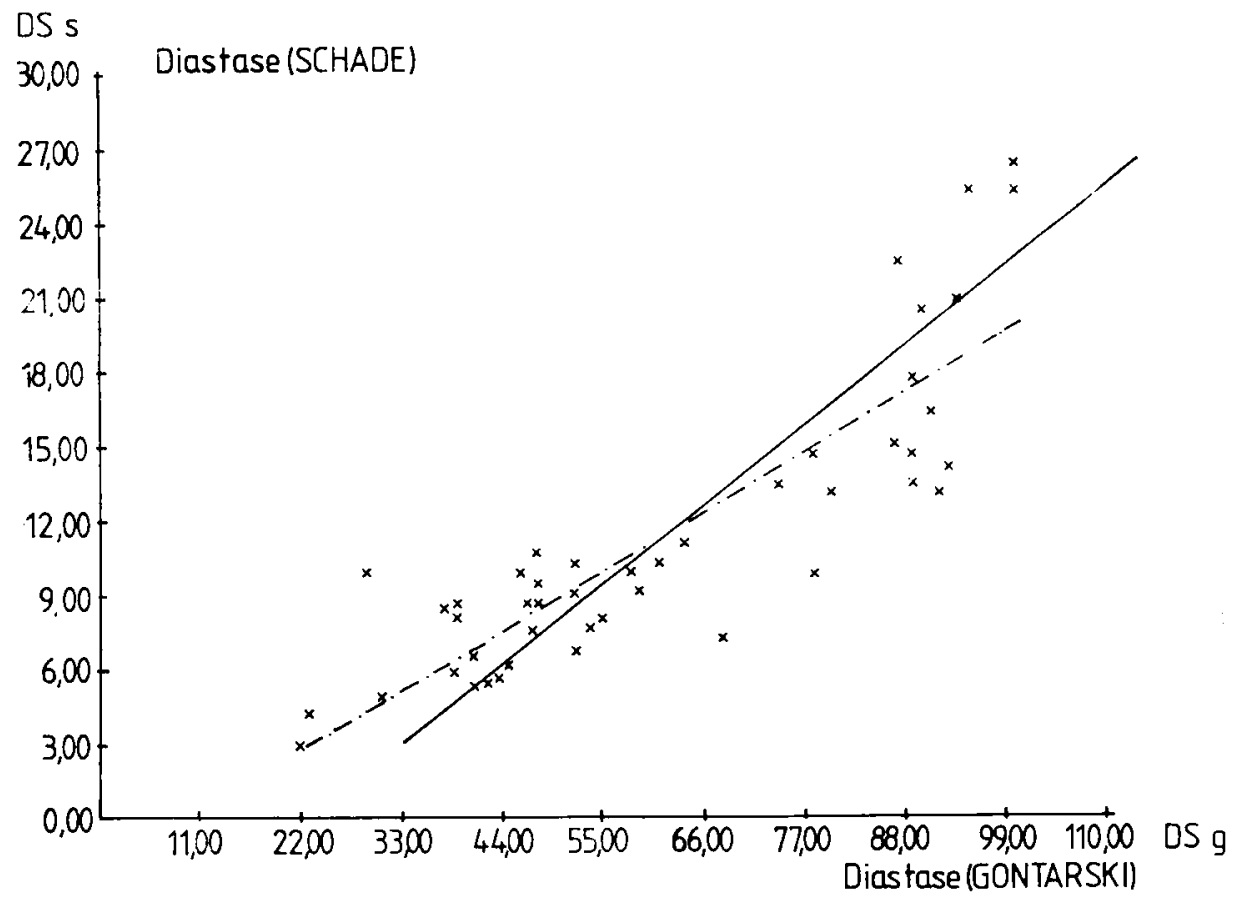

Aвв. 1. - Die beiden Regressionsgeraden für die Diastase-Bestimmungen

- - - - Regressionsgerade zur Schätzung der Diastasezahl nach Schade, aus einer Messung nach GONTARSKI.

Regressionsgerade zur Schätzung der Diastasezahl nach GonTaRSKI, aus einer Messung nach Schade.

Korrelations-Koeffizient $=0,87$.

FIG. 1. - Regression lines for the determination of diastase

-. - Regression line for estimation of diastase after SCHADE out of a measurement after GONTARSKI.

Regression line for estimation of diastase after GoNTARski out of a measurement after SCHADE.

Corr. coeff. $=0.87$.

\subsection{Invertase}

Die beiden Verfahren zur Messung der Invertaseaktivität wurden bei verschiedenen Honigproben auf Reproduzierbarkeit getestet (Abb. 2). Bei der Methode nach GonTarskı wurde aus jeweils fünf Bestimmungen eines Honigs ein Mittelwert von $\mathrm{SZ}=11,6$ mit ciner Standardabweichnug $\mathrm{s}=1,2$, sowie bei einem zweiten Honig ein Mittelwert von $\mathrm{SZ}=33,9$ mit $\mathrm{s}=4,8$ erzielt. Für die Be- 
stimmung mittels p-Nitrophenyl-a $x$-D-glucopyranosid nach Silgenthaler ergab sich im ersten Fall bei einem Mittelwert von $U=39,7$ ein $\mathrm{s}=0,8$, sowic bei der zweiten Honigprobe beim Mittelwert von $U=129,6$ ein $s=0,7$. Hicraus wird ersichtlich, dass die Reproduzierbarkeit bei der Methode Siliginthalez wesentlich besser ausfällt als bei dem Test nach GONTARSKI.

Zwischen beiden Bestimmungen besteht in unserem Labor folgende Beziehung :

U. Sicgenthaler $=11,9+3,6 \times \mathrm{SZ}$ GonTARsKI

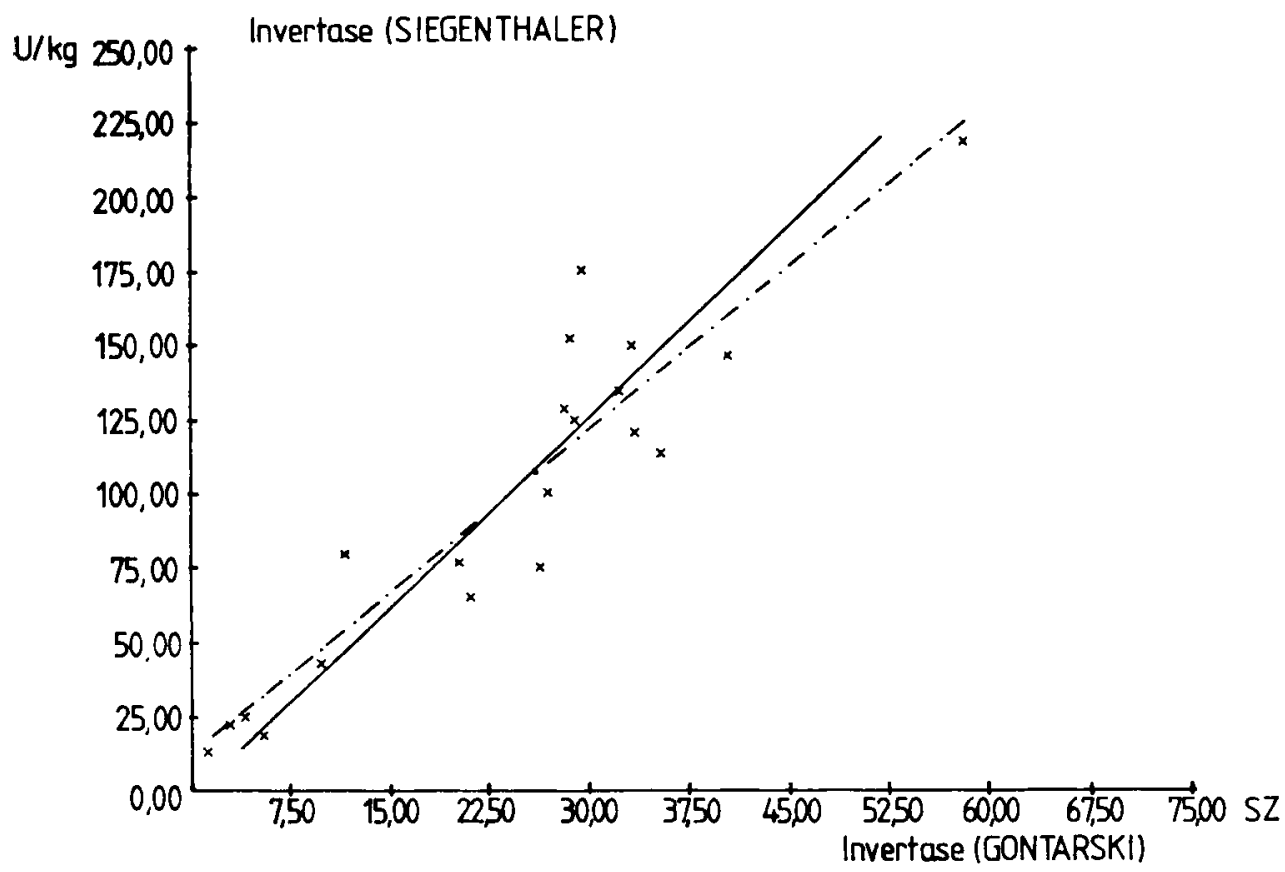

Aвв. 2. - Die beiden Regressionsgeraden fïr die Invertase-Bestimmungen

- - - Regressonsgerade zur Schätzung des Invertasewertes nach Siegenthaler, aus einer Messung nach GonTaRski.

Regressionsgerade zur Schätzung des Invertasewertes nach SiEgENTHALer, aus einer Messung nach Siegenthaler.

Korr. Koeff. $=0,93$.

FIc. 2. - Regression lines for the determination of invertase

- - - Regression line for estimation of invertase after Siegenthaler out of a measurement after GonTakski.

Regression line for estimation of invertase after GONTARSKI out of a measurement after Siegenthaler.

Corr. coeff. $=0.93$. 


\section{3. $H M F$}

Um die Reproduzierbarkeit der HMF-Bestimmung nach WINKLER und der Invertase-Aktivitätsmessung nach SIEGENTHALER vergleichen $\mathrm{zu}$ können, sind für 3 Honige jeweils 10 Bestimmungen durchgeführt worden. Abb. 3 stellt das erzielte Ergebnis dar. Es zeigt sich, dass die Reproduzierbarkeit der Messung der Invertase (U) nach der Methode Siegenthaler viel besser ausfällt als bei einer Bestimmung des HMF-Gehaltes. Dieses gilt vor allem für den Grenzbereich zwischen «zulässig» und «nicht zulässig » gemäss den Bestimmungen zum DIBWarenzeichen.

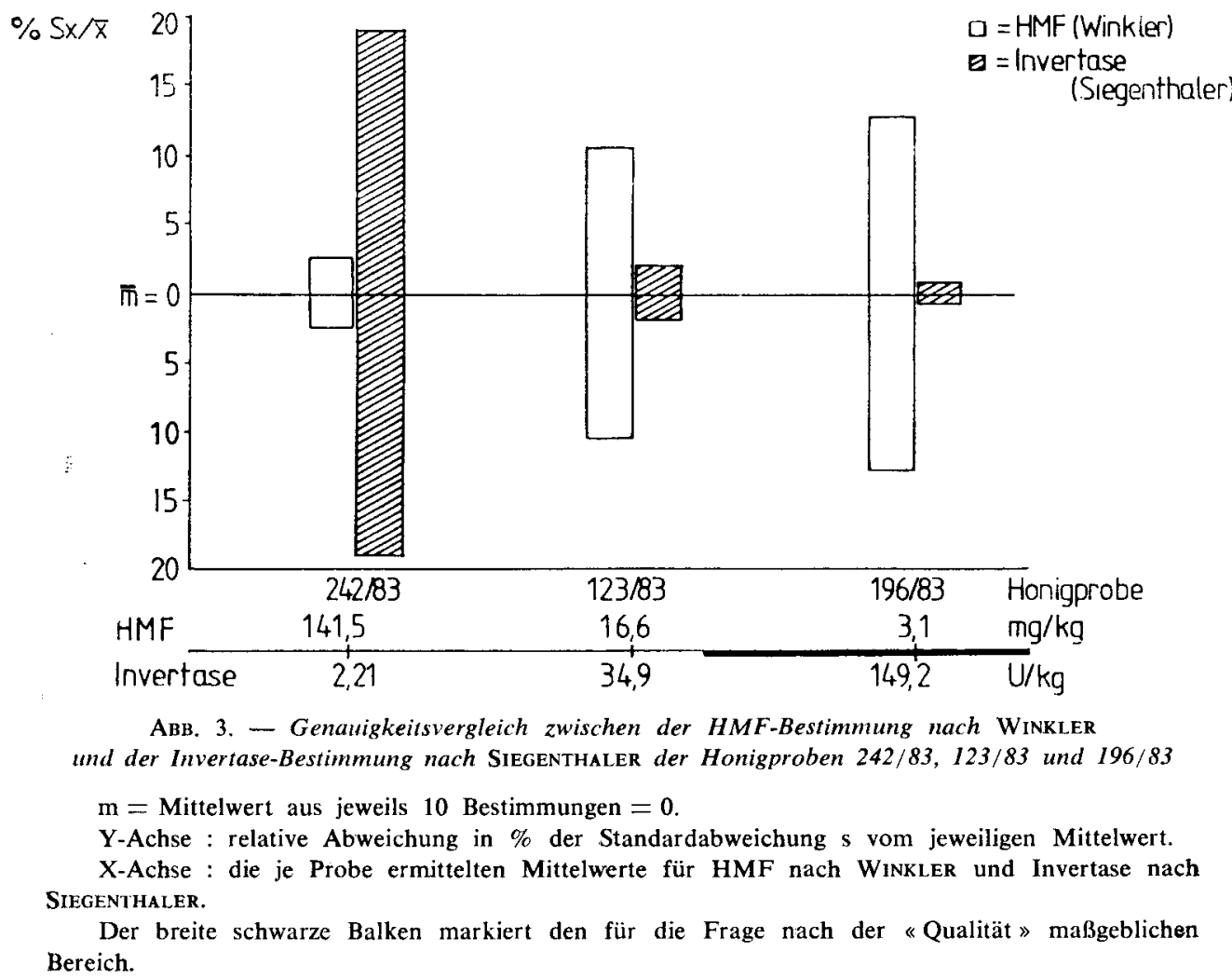

FIG. 3. - Comparison of precision between the determination of HMF after WINKLER and the determination of invertase after SIEGENTHALER from the honey samples 242/83, 123/83 and 196/83

$\mathrm{m}=$ mean of 10 determinations each $=0$.

$\mathrm{Y}$-axis : relative deviation in $\%$ of standard deviation $\mathrm{s}$ from respective mean.

$\mathrm{X}$-axis : mean per sample for HMF after WINKLER and Invertase after SiegenthaleR.

The broad black bar indicates the area which prevails for the question of quality. 
TABI. 2. - Invertase and diastase, after GonTARSki as well as after Siegenthaler resp. SChAdE

TAB. 2. - Invertase und Diastase, sowohl nach Gontarski als nach Siegenthaler bzw. SChade

\begin{tabular}{|c|c|c|c|c|c|}
\hline \multirow[b]{2}{*}{$\begin{array}{l}\text { Probe N:: } \\
\text { Sample No. }\end{array}$} & \multicolumn{2}{|c|}{ Invertase } & \multicolumn{2}{|c|}{ Diastatse } & \multirow[b]{2}{*}{$\begin{array}{l}\text { Pollenanalyse } \\
\text { Pollen analysis }\end{array}$} \\
\hline & $\begin{array}{c}\text { GonTARSKI } \\
\text { SZ }\end{array}$ & $\begin{array}{c}\text { SIEGEN- } \\
\text { THALER } \\
\text { U/Kg }\end{array}$ & $\begin{array}{l}\text { GONIARSKI } \\
\text { DZg }\end{array}$ & $\begin{array}{l}\text { SCHade } \\
\text { DZs }\end{array}$ & \\
\hline B I & 1,4 & 12,72 & 39 & 5,8 & $\begin{array}{l}\text { Südamerika - Mischtracht } \\
\text { South American mixed flow }\end{array}$ \\
\hline B 2 & 9,88 & 43,23 & 48 & 10,6 & $75 \%$ Euphorbia \\
\hline B 3 & 11,68 & 79,79 & 78 & 9,7 & $\begin{array}{l}\text { Waldhonig } \\
\text { Honeydew-honey }\end{array}$ \\
\hline B 4 & 33,26 & 149,24 & 93 & 14,0 & $\begin{array}{l}\text { Kornblumen } \\
\text { Corn-flower }\end{array}$ \\
\hline B 5 & 28,07 & 127,95 & 87 & 14,9 & $\begin{array}{c}49 \% \text { Raps } \\
\text { Rape }\end{array}$ \\
\hline B 6 & 29,31 & 174,04 & 94 & 20.7 & $\begin{array}{l}\text { Wald } \\
\text { Forest }\end{array}$ \\
\hline B 7 & 4,09 & 25,11 & 41 & 5,3 & $\begin{array}{l}\text { Neuseeland - Rata } \\
\text { New-Zealand - Rata }\end{array}$ \\
\hline B 8 & 2,83 & 22,25 & 54 & 7,7 & $\begin{array}{l}\text { Neuseeland - Klee } \\
\text { New-Zealand - Clover }\end{array}$ \\
\hline B 9 & 26,36 & 75,18 & 91 & 16,2 & $\begin{array}{l}\text { Deutschland - Mischtracht } \\
\text { Germany mixed flow }\end{array}$ \\
\hline B 10 & 20,95 & 65,17 & 68 & 7,2 & $\begin{array}{l}\text { Deutschland - Raps } \\
\text { Germany - Rape }\end{array}$ \\
\hline B 11 & 35,34 & 113.01 & 89 & 14,5 & $\begin{array}{l}\text { Deutschland - Raps + Obst } \\
\text { Germany - Rape + Fruits }\end{array}$ \\
\hline B 12 & 28,45 & 151,47 & 89 & 13,3 & $\begin{array}{l}\text { Deutschland - Honigtau } \\
\text { Germany - Honcydew }\end{array}$ \\
\hline B 13 & 26,84 & 99,97 & 78 & 14,5 & $\begin{array}{l}\text { Übersec } \\
\text { Overseas }\end{array}$ \\
\hline B 14 & 28,98 & 123,81 & 89 & 17,6 & $\begin{array}{l}\text { Ubersee } \\
\text { Overseas }\end{array}$ \\
\hline B 15 & 32,20 & 133,99 & 90 & 20,3 & $\begin{array}{l}\text { Übersee } \\
\text { Overseas }\end{array}$ \\
\hline B 16 & 20,14 & 76,45 & 38 & 8,4 & $\begin{array}{l}\text { Ägypten - Citrus } \\
\text { Egypt - Citrus }\end{array}$ \\
\hline B 17 & 5,51 & 18,60 & 64 & 11,0 & $\begin{array}{l}\text { Frankreich - Edelkastanie } \\
\text { France - Sweet chestnut }\end{array}$ \\
\hline B 18 & 33,44 & 120,00 & 97 & 26,1 & $\begin{array}{l}\text { Frankreich - Sonnenblumen } \\
\text { France - Sunflower }\end{array}$ \\
\hline B 19 & 55,96 & 216,95 & 95 & 25,0 & $\begin{array}{l}\text { Waldhonig } \\
\text { Honeydew honey }\end{array}$ \\
\hline B 20 & 40,38 & 145,59 & 69 & 9,8 & $\begin{array}{l}\text { Waldhonig } \\
\text { Honeydew honey }\end{array}$ \\
\hline
\end{tabular}




\subsection{Invertase und Diastase}

Für cinen Teil der unter 3.1 und 3.2 beschriebenen Ergebnisse haben wir beide Doppelbestimmungen durchgeführt. Diese Daten sind einschliesslich der Resultate zur Honigherkunftsbestimmung in Tabelle 2 angegeben.

\section{DISKUSSION}

\subsection{Diastase}

Die deutsche Honigverordnung verlangt für Honig cinen Mindestgehalt an Diastase, entsprechend der Diastasezahl 8 nach Schade $(D Z s=8)$. Mit einer $95 \%$ igen Sicherheit liegen alle Honige, die bei der Diastase-Bestimmung nach GonTARskı einen Wert unter $30 \%(\mathrm{DZg}<30 \%)$ erreichen, unter DZs 8 . Für eine $1 \%$ Wahrscheinlichkeit liegt die Grenze bei $\mathrm{DZg}=20 \%$. Desgleichen gilt, bei $\mathrm{p}=0,05$ muss $\mathrm{DZg}>70 \%$ sein; und bei $\mathrm{p}=0,01 \mathrm{DZg}=80 \%$.

Für die Untersuchungspraxis ergibt sich hieraus, dass nur in den Fällen, in denen die schnellere Diastase-Bestimmung nach GonTarsk I Werte zwischen $30 \%$ und $50 \%$ ergeben hat, die zeitaufwendige Bestimmung nach ScHaDe eingesetzt zu werden braucht. Da in unserem Labor nur eine mangelnde Übereinstimmung der Diastase-Messungen nach Sieginthaler (1975) und Schadi erzielt werden konnte, bevorzugten wir - nicht zuletzt auch aus Kostengründen - das beschriebene Verfahren.

\subsection{Invertase}

In der europäischen Honig-Untersuchungspraxis ist es üblich, von einem Honig, der als «fermentreich» deklariert werden soll, eine Mindestaktivität an Invertase von 10 Einheiten zu fordern (SZ 10).

Werden hierbei - wie in früheren Jahren in Deutschland - Verfahren und Einheiten nach GonTARski angewandt, so erscheint es aufgrund unserer Vergleichsmessungen gerechtfertigt, statt 10 Einheiten nach GonTARSKI die dementsprechenden 40 Einheiten nach Siegenthaler einzusetzen.

Nach HADOrN et al., 1962 stimmen die von Gontarski angegeben Werte zahlenmässig mit seiner Definition der Saccharasezahl überein. Daher erscheint es gerechtfertigt, auch eine entsprechende Umrechnung der Invertase-Einheiten nach Siegenthaler in Saccharase-Zahlen nach HADORN vorzunehmen.

Bei der Korrelation der Saccharasezahl nach GonTARskı mit der Extinktion bei $400 \mathrm{~nm}$ nach SiEgEnTHALER hat sich für die beschriebenen 20 Honige herausgestellt, dass die Beziehung $\mathrm{SZ}=37,47 \mathrm{E}_{\mathbf{4 0 0} \mathrm{um}}+0,6$ gilt. Dieses deutet 
darauf hin, dass möglicherweise, wie von WHITE (1975) augedeutet, die polarographische Methode nicht direkt mit dem reduktometrischen Verfahren verglichen werden kann.

Zahlreiche Autoren haben den Nachweis erbracht, dass der Gehalt an Invertase einen guten Indikator fü etwaige Hitzeschäden im Honig darstellt. HADORN et al. (1962) beurteilen die Invertase sogar als einen empfindlicheren Indikator als den Gehalt an HMF. Eigene Befunde über den Einfluss einer Erwärmung auf den Gehalt an Invertase, Diastase und HMF in Honig (van PraAcil et al. in Vorbereitung) bestätigen diese Aussage. Da für die Messung des HMF-Gehaltes der Einsatz des giftigen p-Toluidin erforderlich ist, - es sei denn man wählt die arbeitsaufwendige Methode nach WHITE, 1979 —, erscheint es angebracht, eine Änderung der derzeitigen Honig-Untersuchungspraxis vorzunehmen, d.h., vorrangig die Messung der Invertaseaktivität als Indikator für Naturbelassenheit und Reife durchzuführen. Anders als das HMF ergibt die Höhe der Invertase-Aktivität auch einen Hinweis, inwieweit das Sammelgut durch die Honigbiene verarbeitet und mit enzymhaltigem Drüsensekret angereichert wurde.

Da die Messung der Invertase-Aktivität nach GONTARskı sehr zeitaufwendig ist, wurde in der Untersuchungspraxis der deutschen Bieneninstitute die HMFBestimmung vorrangig durchgeführt. Ein Vergleich von Zeitaufwand und Kosten der beiden Bestimmungsmethoden (Invertase nach SiEgenthales und HMF nach WINKLER) ergibt nach unseren Erfahrungen keine markanten Unterschiede.

Andererseits belegen die Messungen zur Reproduzierbarkeit (Abb. 3) ein wandfrei, dass die Bestimmung nach SiEgEnTHALER in dem kritischen Bereich (40 Einheiten) deutlich genauer ist als die HMF-Bestimmung bei $15 \mathrm{mg} / \mathrm{kg}$. Auch White (1979) hat eine schlechte Reproduzierbarkeit der WINKI.ERBestimmung festgestellt und nicht zuletzt deshalb ein anderes Meßverfahren (Direkte Absorption bei 284 und $336 \mathrm{~nm}$ ) vorgeschlagen.

Um den Einsatz des giftigen p-Toluidin zu beschränken, schlagen wir den Einsatz der Invertase-Bestimmung nach SiEgenthaler als erstes Kriterium für die Naturbelassenheit vor. Der HMF-Test sollte zweitrangig eingesetzt werden, insbesondere dann, wenn lebensmittelrechtliche Belange anstehen oder von Natur aus enzymschwache Honige vorlicgen (z.B. Robinie). Es wäre dann die Bestimmung nach WHITE der nach WINKLER vorzuziehen. Hierdurch wäre eine genauere und im Hinblick auf das Kriterium «Naturbelassenheit» sachgerechtere Analyse verfügbar, um einen Verstoss gegen die Warenzeichenbestimmungen des DIB feststellen zu können.

Als Beispiel sei folgendes Zahlenverhältnis aufgeführt : Honige, die einen Invertase-Wert (U) von weniger als 36 aufweisen, liegen mit 1,3\% Wahrscheinlichkeit unter den zu verlangenden 40 Einheiten. Eine gleiche Zuverlässigkeit $(\mathrm{p}=0,013$ ) bei der HMF-Bestimmung liegt erst bei $20,2 \mathrm{mg} / \mathrm{kg}$ HMF vor. Erst 
bei diesem Wert kann man sicher sein, dass der HMF-Gehalt über $15 \mathrm{mg} / \mathrm{kg}$ liegt. Erst durch mehrfaches Messen von Honigen mit einem HMF-Gehalt zwischen 10 und $20 \mathrm{mg} / \mathrm{kg}$ kann die Zuverlässigkeit der Ergebnisse erhöht werden. Bei der Invertasebestimmung brauchen nur Honige mit Werten zwischen $\mathrm{U}=36$ und $U=44$ mehrfach gemessen werden, um $p=0,01 \mathrm{zu}$ erreichen.

Eingegangen im März, 1984.

Angenommen im Dezember 1984.

\section{RESUME}

DETERMINATION DE LA DIASTASE, DE L'INVERTASE ET DE L'HYDROXYMETHYLFURFURAL DANS LE MIEL

Il existe diverses méthodes courantes d'analyse chimique afin de déterminer si le miel a conservé son caractère naturel ou s'il a été abîmé par la chaleur et le stockage. Nous avons mesurć la précision et le temps nécessairc pour un certain nombre d'entre elles.

On montre que la détermination de lactivité de la saccharase (invertase, $\alpha$-glucosidase) par Siegenthaler présente de nets avantages comparée à la méthode usuelle de détermination de l'hydroxymethylfurfural (HMF), utilisée par WINKLER. La précision et la reproductibilité des valeurs de la saccharase sont plus élevées, la manipulation du réactif toxique Toluidin n'est pas nécessaire et le temps passé est à peu près le même pour les deux méthodes.

Puisque le degré d'activité de la saccharase est habituellement un meilleur indicateur, que la teneur en HMF, de la conservation naturelle du miel, nous proposons d'utiliser l'activité de la saccharase comme premier critère de la conservation naturelle du miel dans le contrôle courant de la qualité du miel - tel que c'est pratiqué par la Fédération des Apicultcurs Allemands. L'analyse de la teneur en HMF ne devrait être utilisée que pour les miels qui ont naturellement une faible teneur en enzymes.

Il existe une relation linéaire entre la détermination de la saccharase par Siegenthaler et celle par Gonrarski, ce qui autorise une simple conversion des unités respectives.

\section{SUMMARY}

\section{DETERMINATION OF DIASTASE, INVERTASE AND HYDROXYMETHYLFURFURAL IN HONEY}

In order to find out if honey is preserved in its natural condition or damaged by heat and storage, various methods of chemical analyses are common. We measured the accuracy and time required for some of them.

It was shown that the determination of saccharase activity (invertase, $a$-glucosidase) by SiEgenthaler has eminent advantages compared to the common method of hydroxymethylfurfural (HMF) determination used by WINkLER. The accuracy and reproductibility of saccharase values are higher, handling the poisonous reagent Toluidin is not necessary and the time of testing is nearly equal for both methods.

Because the grade of saccharase activity usually is a better indicator for natural preservation of honey than the HMF content, we propose for the general practice of honey quality control 
- as used by the German Beekeepers Federation - to determine saccharase activity as first criterion for natural preservation of honey. Analysis of HMF content should be used in those honey samples only which are low in enzyme content by nature.

There is a linear relation between saccharase determination by SiEgenthal.er and by GonTARSK1, which allows a simple conversion of respective units.

\section{LITERATUR}

DustmanN J.H., 1967. - Messung von Wasserstoffperoxid und Enzymaktivität in mitteleuropäischen Honigen. Z. Bienenforsch., 9 (2), 66-73.

Gontarski H., 1954. - Eine elektrophotometrische Halbmikromethode zur quantitativen Diastasebestimmung im Bienenhonig. Z. Lebensm. Unters.-Forsch., 98 (3), 205-213.

Gontarski H., 1957. - Eine Halbmikromethode zur quantitativen Bestimmung der Invertase im Bienenhonig. Z. Bienenforsch., 4 (2), 41-45.

Gontarskı H., 1961. - Über den Einfluss von Wassergehalt und pH des Honigs auf den Grad der Wärmeschädigung der Invertase. Z. Bienenforsch., 5 (7), 191-198.

HadorN H., 1961. - Zur Problematik der quantitativen Diastasebestimmung in Honig. Mitt. Geb. Lebensmittelumers. Hyg., 52 (2), 67-103.

Hadorn H., Zïrcher K., Doevelaar F.H., 1962. - Über Wärme und Lagerschädigungen von Bienenhonig. Mitt. Geb. Lebensmittelunters. Hyg., 53 (3), 191-229.

Kerkvi.ier J.D., A.P.J. v.d. Putten, 1973. - The Diastase Number of Honey : A Comparative Study. Z. Lebensm. Unters.-Forsch., 153, 87-93.

Schade J.E., Marsh G.L., Eckert J.E., 1958. - Diastase Activity and Hydroxy-methyl-furfural in Honey and their Usefullness in Detecting Heat Alteration. Food Res., 23, 446-463.

Siegenthaler U., 1975. - Bestimmung der $\alpha$-Amylase im Bienenhonig mit einem handelsüblichen, farbmarkierten Substrat. Mitt. Geb. Lebensmittelunters. Hyg., 66, 393-399.

Siegenthaler U., 1977. - Eine einfache und rasche Methode zur Bestimmung der $\alpha$-Glucosidase (Saccharase) im Honig. Mitt. Geb, Lebensmittelunters., 68, 251-258.

White J.W., Kushnir I., Subers M.H., 1964. - Effect of Storage and Processing Temperatures on Honey Quality. Food Technol., 18 (4), 153-156.

White J.H. Jr., 1975. - Composition of Honey. In : Honey - A comprehensive survey. Ed. E. Crane; Heinemann : London.

White J.W. Jr., 1979. - Methods for Determining Carbohydrates, Hydroxymethylfurfural and Proline in Honey : Collaborative Study. J. Assoc. Off. Anal. Chem., 62 (3), 515-526.

Winkler O., 1955. - Beitrag zum Nachweis und zur Bestimmung von Oxymethylfurfurol in Honig und Kunsthonig. Z. Lebensm. Unters.-Forsch., 102 (3), 161-167. 